We try to publish authors' responses in the same edition with readers' comments. Time constraints might prevent this in some cases. The problem is compounded in a bimonthly journal where continuity of comment and redress are difficult to achieve. When the redress appears 2 months after the comment, 4 months will have passed since the article was published. Therefore, we would suggest to our readers that their correspondence about published papers be submitted as soon as possible after the article appears.

\section{LEEP in the Family Practice Setting}

To the Editor: I am writing to express my concerns about the conclusions in the article by Lyman and Morris about the use of the loop electrical excision procedure (LEEP) (Lyman DM, Morris B. LEEP in the Family Practice Setting. J Am Board Fam Pract 2003;16:204-8). The authors conducted a retrospective study of an underserved rural community in the management of cervical intraepithelial neoplasia (CIN) 2 and 3. They felt that their experience justified the use of LEEP. After reading the article, my concern is whether LEEP was medically necessary and whether it would have been more appropriate to offer cryotherapy for their patients. There is no substantive difference in outcomes between LEEP and cryotherapy regarding persistence and resolution in the treatment of squamous intraepithelial lesions for lowgrade lesions, assuming a negative endocervical curettage. ${ }^{1}$ Cryotherapy can be provided at a lower cost and has fewer adverse complications than LEEP.

Jim Nuovo, MD

University of California, Davis

\section{Reference}

1. Nuovo J, Melnikow J, Willan AR, Chan BK. Treatment outcomes for squamous intraepithelial lesions. Int J Obstet Gynecol 2000;68:25-33.

The above letter was referred to the authors of the article in question, who offer the following reply.

To the Editor: Dr. Nuovo raises questions about the appropriateness of using LEEP to treat CIN 2 and CIN 3 when cryotherapy may offer a less expensive and equally efficacious treatment. There are several reasons why cryotherapy is an inferior treatment for high-grade CIN.

One, with no specimen to submit for histologic examination, both laser vaporization and cryotherapy will miss the unsuspected adenocarcinoma in situ and microinvasive squamous cell carcinoma found in $2 \%$ to $3 \%$ of specimens. $^{1,2}$

Two, complications are not necessarily fewer with cryo. Mitchell and colleagues ${ }^{3}$ found no differences in rates of complications (or success) when they randomized treatment to 1 of 3 modalities - cryo, laser, or LEEP. To state that cryo has "fewer complications" is simply not true when treatment is stratified by squamous intraepithelial lesion (SIL) grade, endocervical gland involvement, and lesion size-as was done by Mitchell and colleagues. $^{3}$

Three, it may be more expensive to treat CIN with cryo if we are to believe the work of Sparks and colleagues. ${ }^{4}$ Fifty-one of 83 women $(61.4 \%)$ treated with cryo had an "inadequate" follow-up colposcopy versus 5 of 24 women $(20.8 \%)$ treated with LEEP or cold-knife conization (CKC) [Note that the terms "inadequate" and "adequate" were not defined. I assume they are equivalent to the more familiar terms "unsatisfactory" and "satisfactory." A "satisfactory" colposcopic examination is one in which both the entire squamocolumnar junction (SCJ) and the upper limits of the lesions are visualized.] Their conclusion was that treatment of CIN with cryotherapy increased the risk of "inadequate" follow-up colposcopy. Because the current management of an "unsatisfactory" (and I assume "inadequate") colposcopy is to proceed to LEEP or CKC, the authors posit that an unknown number of LEEPs are done for this reason. The total cost of cryo followed by LEEP is unknown but not insignificant.

Four, Harper and colleagues ${ }^{5,6}$ in 2 papers describe the significant symptoms experienced during the healing process after cryotherapy. The authors documented a more prolonged, malodorous, and painful healing process than had been previously recognized, although they alluded to the need to reproduce this study in post-LEEP women. My experience is clearly unscientific, but when I ask women who have had both procedures their preference, they have all stated that LEEP was less painful with fewer side effects than the preceding cryosurgery.

In summary, cryotherapy is a reliable, easy-to-use, low-cost tool. However, loop electrical excision of the transformation zone or LEEP as described above is a superior treatment for CIN. The technique has a proven safety record, is easily learned by the family physician, and the equipment-which can also be used for dermatology surgery-is becoming more affordable. New recommendations on the use of LEEP are forthcoming and may resolve unanswered questions about the most appropriate treatment of epidemic CIN.

David Lyman, MD, MPH UT/Jackson Madison County General Jackson, Miss.

\section{References}

1. Wright TC Jr, Gagnon S, Richart RM, Ferenczy A. Treatment of cervical intraepithelial neoplasia using the loop electrosurgical excision procedure. Obstet Gynecol 1992; $79: 173-8$. 
2. Ferenczy A, Choukroun D, Arseneau J. Loop electrosurgical excision procedure for squamous intraepithelial lesions of the cervix: advantages and potential pitfalls. Obstet Gynecol 1996;87:332-7.

3. Mitchell MF, Tortolero-Luna G, Cook E, Whittaker L, Rhodes-Morris H, Silva E. A randomized clinical trial of cryotherapy, laser vaporization, and loop electrosurgical excision for treatment of squamous intraepithelial lesions of the cervix. Obstet Gynecol 1998;92:737-44.

4. Sparks RA, Scheid D, Loemker V, et al. Association of cervical cryotherapy with inadequate follow-up colposcopy. J Fam Pract 2002;51:526-9.

5. Harper DM, Mayeaux EJ Jr, Daaleman TP, Woodward LD, Ferris DG, Johnson CA. The natural history of cervical cryosurgical healing. The minimal effect of debridement of the cervical eschar. J Fam Pract 2000;49:694-700.

6. Harper DM, Mayeaux EJ Jr, Daaleman TP, Johnson CA. Healing experiences after cervical cryosurgery. J Fam Pract 2000;49:701-6. 